\title{
LIGHT \\ COSMIC MESSAGES FROM THE PAST
}

Michael C. Wiescher ${ }^{1}$ and K. Langanke ${ }^{2}$ - DOI: 10.1051/epn/2015403

1 University of Notre Dame and JINA - Notre Dame, IN 46556, USA

II GSI Helmholtzzentrum für Schwerionenforschung

und Technische Universität Darmstadt - Darmstadt, Germany

The universe is filled with electromagnetic radiation, and visible light covers only a small section of its spectrum.

The dominant sources of light are stars, with the energy

originating from nuclear fusion processes in their

interior. The question of energy generation in

our sun and in other stars is the main focus

of nuclear astrophysics.
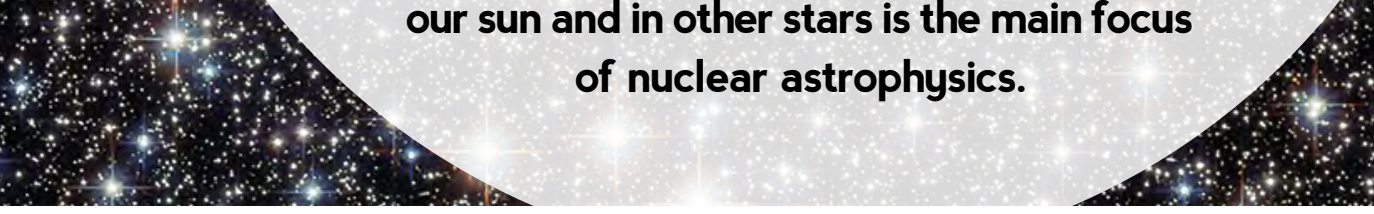

(1)

he field of nuclear astrophysics addresses the nature of the nuclear reactions, and reaction sequences that generate stellar energy and drive the evolution of stellar life from birth to death. The field also addresses the origin of the elements, during the evolution of our universe from its beginning 13.7 billion years ago to the present time. Nuclear reaction processes represent the engine for the evolution of stars and the chemical evolution of our universe. The light from distant stellar objects provides us with the information that tests the theories and guides the experiments of the nuclear astrophysics community. Thus light observed today allows us to look back into the past and to unravel the evolution of our universe. In this article we provide an overview of selected astrophysical sources of light and what they tell us about the history of our universe.

\section{Light from our Sun}

Without light from our sun and far distant stars, the earth would be cold and dark. Our sun provides the light and heat that drive the evolution of our planet, it is the key to climate evolution, and provides the energy for most of the geo-chemical and bio-chemical processes that make earth a habitable planet. Light is radiated from the outer atmosphere - the photosphere - of the sun, having an average temperature of about $5500^{\circ} \mathrm{C}$. The energy heating the solar surface is, however, generated deep in the solar interior through nuclear fusion and is being transported by radiative and convective processes to the solar surface. The light reaches earth with the characteristic Planck blackbody distribution from the ultraviolet to the infrared

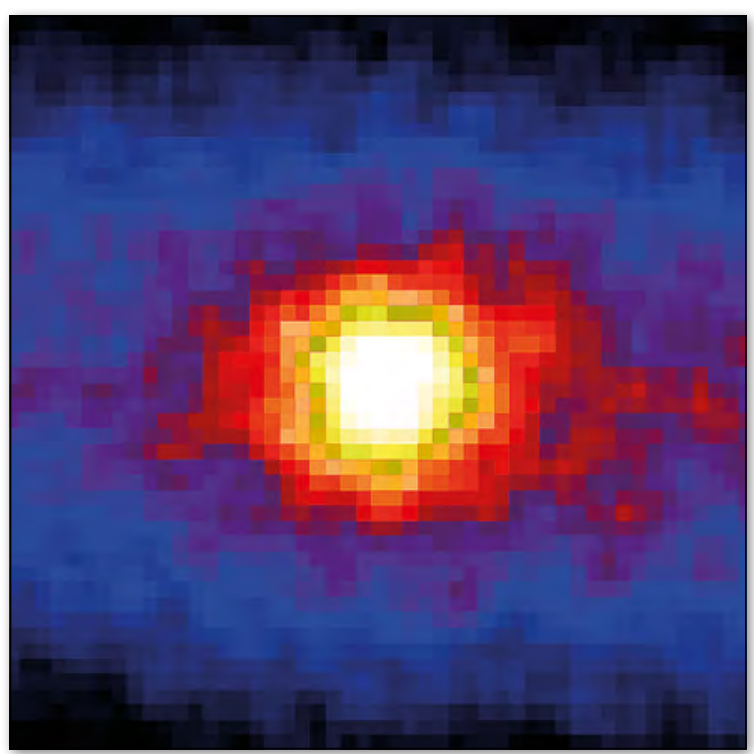

$\triangle$ The crammed centre of Messier 22. This image shows the centre of the globular cluster Messier 22 also known as M22, as observed by the NASA/ESA Hubble Space Telescope. Globular clusters are spherical collections of densely packed stars, relics of the early years of the Universe, with ages of typically 12 to

13 billion years. (c) ESA/Hubble \& NASA

4 FIG. 1:0ur sun observed in neutrinos. Image Credit: http://apod. nasa.gov/apod/ ap980605.html 
$\nabla$ FIG. 2: The Cosmic Microwave Background Radiation as observed by the COBE satellite constitutes a perfect blackbody spectrum with temperature $T=2.725 \mathrm{~K}$. Image Credit: Wikipedia range of the electromagnetic spectrum, peaking in the wavelength range of visible light. Evolution has made our eyes, our main organs for detecting electromagnetic radiation, most sensitive to exactly this range of visible light transmitted by the sun to our planet.

Besides light, the second messenger indicating the existence of solar fusion reactions are neutrinos. These weakly interacting neutral particles are being produced in enormous quantities by weak interaction processes in the core of the sun. Unlike electromagnetic radiation, neutrinos can penetrate matter without much interaction and reach earth with (nearly) the speed of light. They are measured by deep underground neutrino detectors, typically large vessels filled with water or scintillator liquids, which detect a few of the enormous flux of neutrinos that daily penetrate earth (Fig. 1).

Through the intensity of solar light emission and the measurements of solar neutrino flux we have obtained a detailed understanding of the nuclear reaction processes that power not only our sun but also the earth as part of the planetary system [1]. The main energy source consists of the pp-chains (proton-proton chains): a sequence of nuclear reactions fusing four protons into helium $(4 \mathrm{He})$, under the release of energy in the form of photons, neutrinos, and the kinetic energy of other reaction products. The first reaction in the sequence is the extremely slow $\mathrm{p}+\mathrm{p} \Rightarrow \mathrm{d}$ conversion from two protons to a deuteron. As this reaction is based on the weak interaction, which converts one proton into a neutron, it is very slow and causes the long life of our sun. The various reactions associated with the pp-chains are being studied in deep underground laboratories in order to determine the lifespan of our sun and the amount of luminosity our sun will produce over the next few billion years before running out of hydrogen fuel.

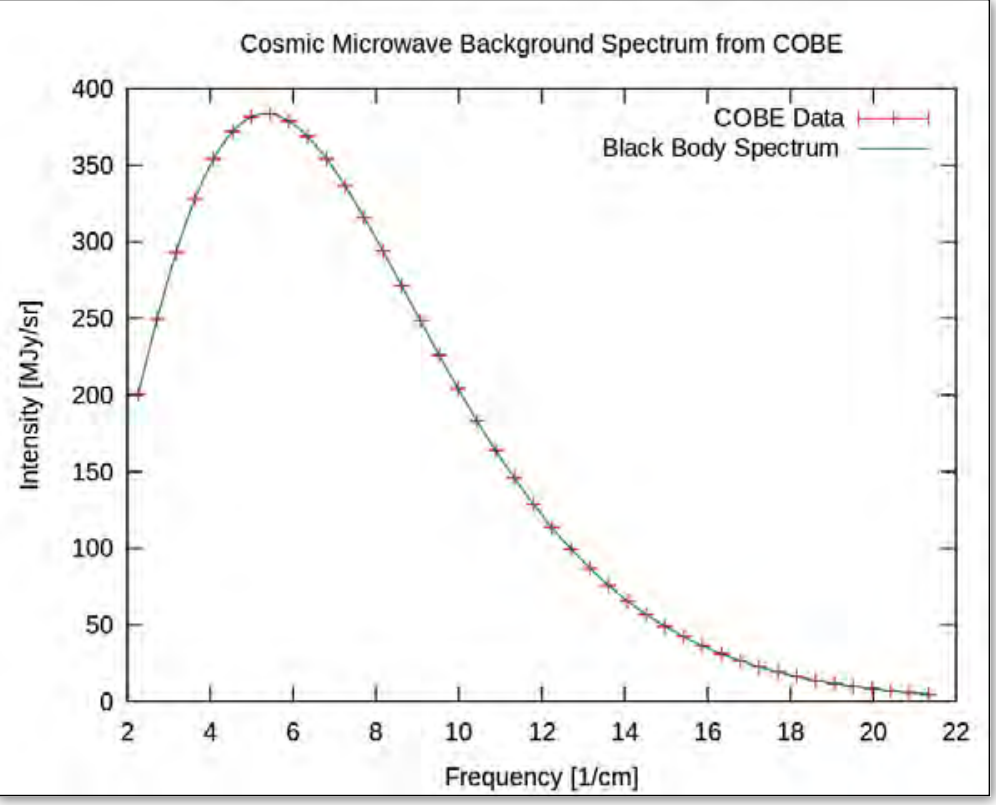

\section{Light in the early universe}

The dominant source of photons in our universe is the Big Bang, which took place about 13.7 billion years ago and is considered to be the birth of our rapidly expanding universe. The early universe was filled with different kinds of matter, including free protons and neutrons. Dominant components were photons, intense electromagnetic radiation that cooled with the expansion. After the first minute the energy of the photons was reduced to a sufficiently low level, allowing deuterium nuclei, which were continuously formed by fusion of protons and neutrons, to survive against photodisintegration. This initiated primordial nucleosynthesis, forming nuclei up to helium and lithium. After 400 Million years of expansion the photon energy was reduced further to the electronvolt $(\mathrm{eV})$ level, insufficient for atomic ionization and excitation processes. Photons and baryons decoupled, and the first generation of stars was formed. Photons cooled further to ever expanding wavelengths until today, when the universe has cooled to a temperature of 3 Kelvin. These photons are visible with radio telescopes as $3 \mathrm{~K}$ background radiation. The structure and distribution of this radiation provides insight into the early Big Bang development [2] (Fig. 2).

\section{Light of early stars}

Over the last decade remarkable results have been obtained in the discovery and spectroscopic analysis of light of early-generation stars. These stars emerged through gravitational contraction about 13 billion years ago, after the photons and baryons decoupled. In their interior these first stars offered the first site with sufficient temperature and density conditions to allow for nuclear reactions that form heavier elements. The respective nucleosynthesis was driven by the pp-reactions on the hydrogen-helium fuel that was provided by the Big Bang. With the contraction of the stellar core, helium burning by fusion between three helium nuclei - the triple alpha process - becomes possible. It forms ${ }^{12} \mathrm{C}$ and by subsequent alpha-capture ${ }^{16} \mathrm{O}$, the two most critical elements for the development of biological life [3]. Indeed, recent spectroscopic analysis - based on Hubble or other space or ground based observatories - of the light that reaches us from these first stars indicates primordial elements as well as carbon and oxygen abundance (Fig 3 ). These observations provide a story of the first origin of carbon and oxygen, key elements and stepping stones for subsequent nucleosynthesis patterns that emerged in the following generations of stars.

\section{Light from old metal-poor stars}

Stellar light provides a tantalizing glimpse on the unique origin of heavy elements. The spectroscopy of light from old stars indicates only very few heavy elements. These stars are named metal-poor. Large 

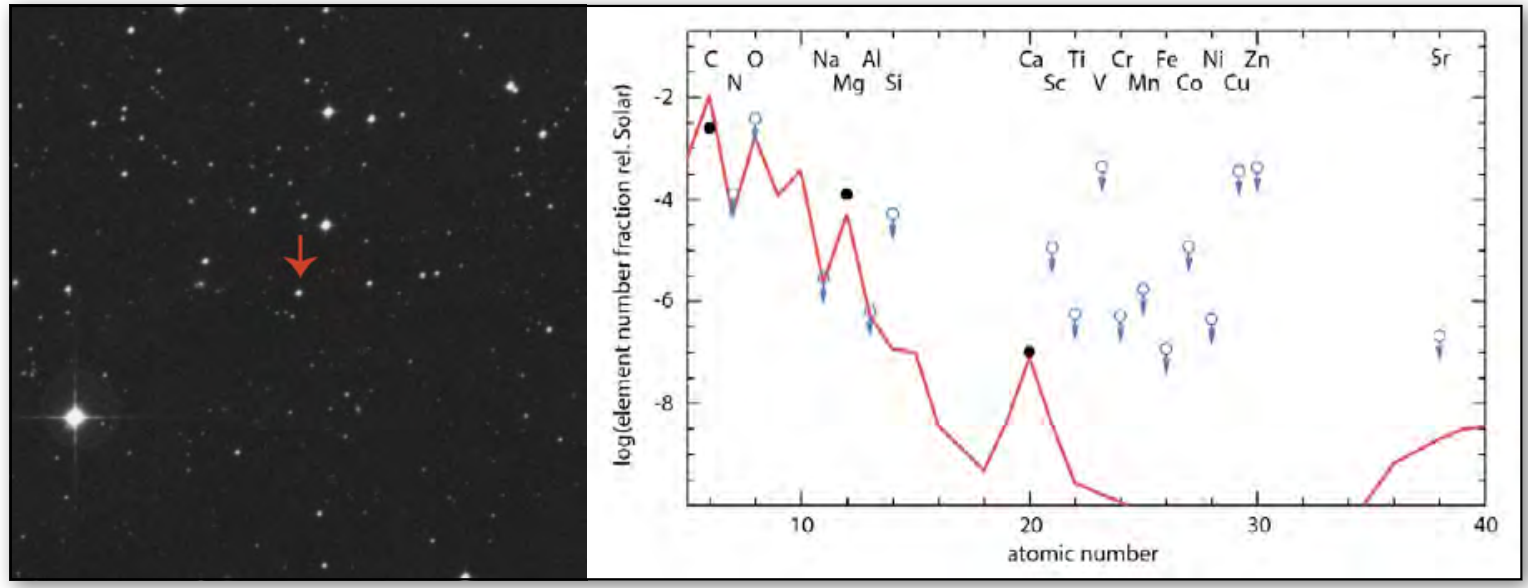

4FIG. 3:

The oldest known star

(arrow in left picture)

with an age of about

13.6 billion years is

located in our Milky

Way at a distance

of 6000 light years

from the sun [4]. Its

abundance pattern

(right picture)

proves early stellar

nucleosynthesis of

light elements like

carbon and oxygen,

galactic surveys of thousands of stars have helped to identify a fairly large number of metal-poor stars. High-resolution spectroscopy of these stars revealed an abundance distribution pattern of heavy elements from about barium to lead and uranium that closely mapped the so-called r-process abundance pattern of our sun (Fig. 4). This is often viewed as proof that there is one unique site for $r$-process production of the heavy elements, with other sites contributing to lower-mass elements.

The r-process or rapid neutron capture process is one of the main sources for heavy element production in our universe. The $\mathrm{r}$-process is envisioned as a process taking place in stellar explosions, which releases a large flux of neutrons that rapidly (within a second) convert the existing abundance distribution of the star into heavy elements. The particular structure of the r-process abundance pattern is closely correlated with the shell structure of massive nuclei and is one of the prime signatures for the importance of microscopic nuclear effects impacting the chemical evolution of the universe. The nuclei encountered during r-process nucleosynthesis are exotic with large neutron excesses and have to be artificially produced in the laboratory to determine their properties. To achieve this goal, large radioactive beam facilities such as FAIR in Germany and FRIB in the United States are currently being built. They will provide the refined nuclear data needed to identify the astrophysical site of the r-process which is currently unknown [6]. For decades supernovae were considered to be the prime candidate. However, recent supernova simulations indicate that this site is not likely. Merging neutron stars are presently viewed as a very suitable site for the r-process revealing astrophysical conditions that seem to match those required for $r$-process nucleosynthesis to produce the observed abundance patterns. The collision and merging of two neutron stars is, however, not a particularly likely event and it is not clear yet whether they have occurred frequently enough to explain the amount of heavy elements observed in the old stars.

\section{Light and stellar evolution}

Stars are traditionally classified by their light characteristics - the absolute luminosity or magnitude and the colour or spectroscopic classification - ranging from blue to red. Sorting each star according to these two parameters gives the so-called Hertzsprung-Russell (HR) diagram (Fig. 5). Most of the stars line up in a diagonal line stretching from bright blue stars to dim red stars. Our sun is in the yellow range. This is the main-sequence of the HR diagram. Other stars cluster in two areas: red bright stars which represent the red giant (RG) branch in the diagram and bright stars stretching over the colour range from blue to red, the so-called asymptotic giant branch (AGB) stars. There are also a number of dim white-bluish stars, which resemble the white dwarfs (WD) branch in the HR diagram. While this diagram was originally only developed for classification reasons, it was later realized that each of these branches corresponds to an evolutionary stage of stellar life. Main sequence stars are in the first phase of hydrogen burning. For lower mass stars such as our sun, this fusion process is facilitated by the pp-chains, described above. For more massive stars, the pp-chains do not produce enough energy to stabilize the star against gravitational contraction and the $\mathrm{CNO}$ cycle, which is based on a sequence of proton capture processes on carbon, nitrogen, and oxygen as catalytic elements, becomes

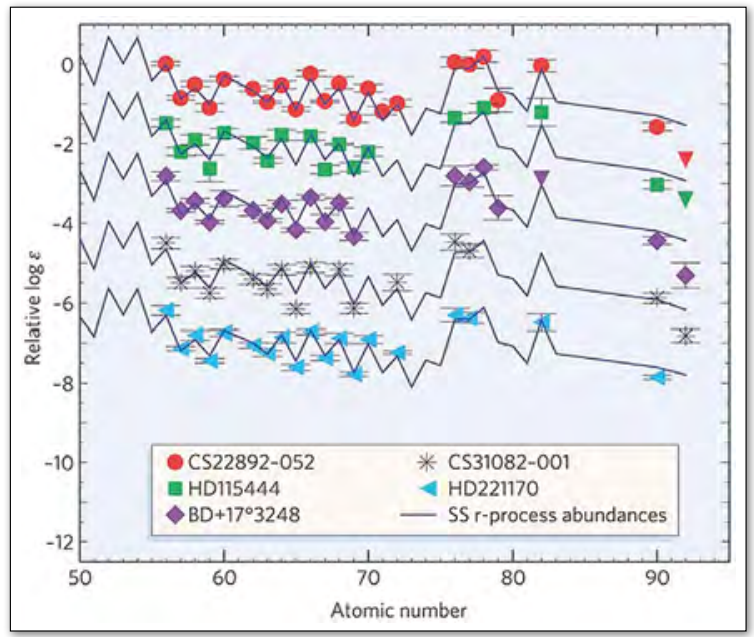

4 FIG. 4:

Abundance pattern of heavy elements in old, metal-poor stars compared to the relative solar $r$-process distribution (solid lines) [5], where $r$-process stands for rapid neutron capture process. The absolute scales have been chosen arbitrarily for better presentation. 
v FIG. 5:

The HertzsprungRussell diagram

displays the

luminosity of a star against its colour

(spectral class).

The location in the

diagram provides

information about

the mass of a star and characterizes

the evolutionary

phase on its journey

through life. Image

Credit: Wikipedia dominant. The $\mathrm{CNO}$ cycle provides only a few percent to the energy generation of our sun, but dominates the energy production in more massive main sequence stars such as the well-known Sirius in Canis Major, Vega in Lyra, and Spica in the Virgo constellation.

When hydrogen fuel is exhausted, the stellar core that contains mainly helium as ashes of the hydrogen burning, contracts under its weight and the hydrogen burning zone expands outward, causing an expansion of the outer layers. This shifts the colour of the star towards red and it emerges as Red Giant. The contraction of the core continues until the temperature and density condition in the center of the star are sufficiently high to trigger helium burning. An example of a red giant in the helium burning phase is Betelgeuse in Orion, a star that is suspected to be close to the end of its helium burning phase. This end occurs when all of the helium in the core is converted to carbon and oxygen. Further energy production comes from the fusion reaction of these two nuclei, followed by a number of subsequent rapid evolution phases that convert these elements by a number of characteristic burning processes to iron and nickel in the stellar core. Stars in these final phases of their life show up as AGB or giant stars in the HR diagram.

The nuclear reactions that determine the stellar energy production, lifetime, and the development of chemical composition have extremely low cross sections, that need to be determined with high accuracy at stellar energy conditions. Enormous progress has been made over the last decade in developing new techniques for these studies, but many critical questions remain primarily associated with the nuclear

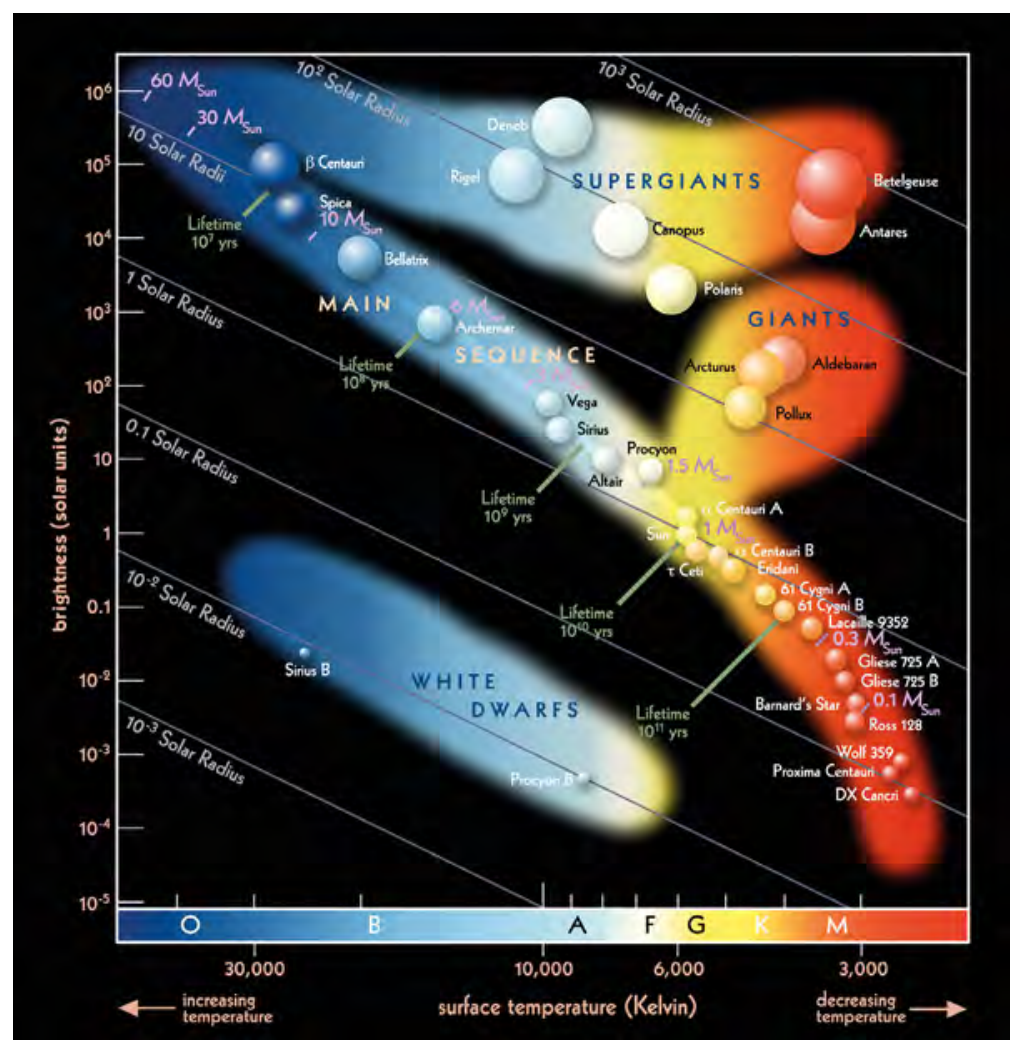

processes at the final stages of stellar life [7]. The main obstacle is that reliable measurements are not only handicapped by extremely low cross sections but also by large amounts of detector background, due to cosmic-ray interaction with the detector material. This limits the experimental range to energies that are well above the stellar energy range. Their study at stellar energies is presently being pursued at the LUNA accelerator that is located deep underground in the Gran Sasso laboratory in Italy, exploiting the large background shielding by the rocks of the Apennine Mountains.

\section{Light from stellar explosion}

Classical and medieval astronomers observed stars which suddenly developed enormous brightness; they called these stars novae or supernovae (new stars). Today, astronomers observe supernovae in far distant galaxies, with a luminosity that parallels the luminosity of the entire host galaxy. Supernovae (SN) are classified by their spectroscopic appearance and are sorted (roughly speaking) in two main classes: SN type I, by the lack of hydrogen lines in the spectrum of the emitted light, and SN type II which does show hydrogen lines. While the sub class SN type Ia is considered to be a gigantic thermonuclear explosion of a star that is triggered by accretion processes in a white dwarf double star system, SN type II basically represents the violent death of a massive star as a consequence of the gravitational collapse of the stellar core [8].

Associated with the rapid energy release in a supernova explosion is an enormously high emission of light or luminosity over the entire electromagnetic spectrum within seconds. The light emission associated with the actual explosion rapidly declines, but multiple new radioactive elements such as ${ }^{56} \mathrm{~N}$ and ${ }^{44} \mathrm{Ti}$ have been formed as a consequence of the nuclear processes occurring during the explosion, and the associated decay energy is slowly released with the characteristic nuclear halflives. The time correlation between the luminosity and the radioactive decay has been particularly observed for the light curve of recent supernovae such as SN 1987a, which is associated with the violent death of the blue supergiant star Sanduleak in the Tarantula Nebula of the Large Magellanic Cloud. The light curve matches successively the decay of ${ }^{56} \mathrm{~N}$, its daughter nucleus ${ }^{56} \mathrm{Co}$ and other radioisotopes that are being produced in the explosion. These observations are mirrored by studies on other supernova remnants such as Cas A in the constellation Cassiopeia, that was first observed in 1680. For Cas A satellite-based X-ray and $\gamma$-ray observatories directly observed the characteristic $\gamma$-lines associated with the decay of ${ }^{44} \mathrm{Ti}$ and delivered direct evidence for the existence of this relatively short-lived isotope [9]. Mapping the sky with $\gamma$-telescopes such as INTEGRAL reveals the image of a radioactive universe resulting from the slow radioactive decay processes associated with supernova remnants along the galactic plane of our Milky Way (Fig. 6). 


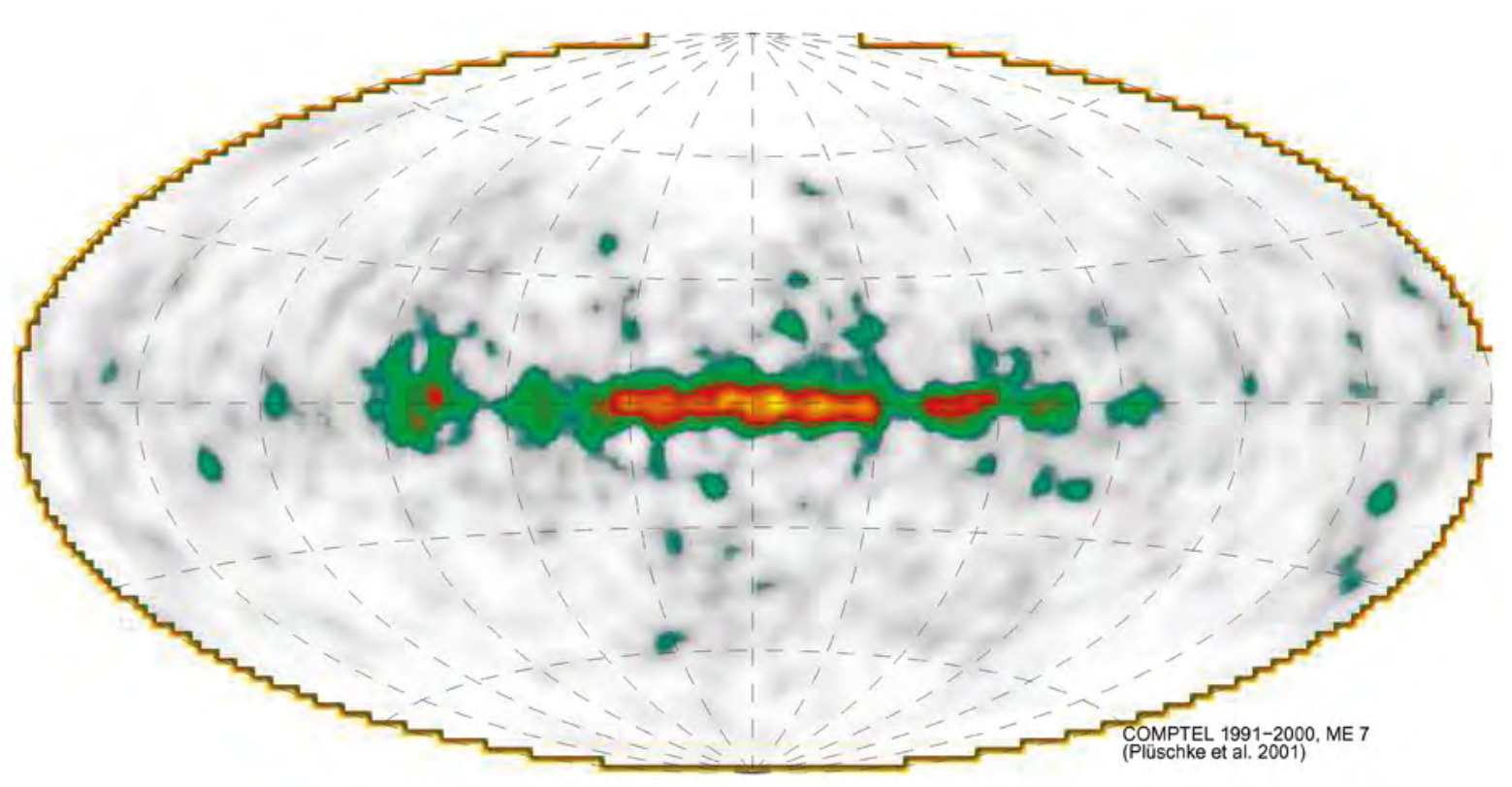

4 FIG. 6: All-sky map of ${ }^{26} \mathrm{Al}$ activities as observed by the COMPTEL and INTEGRAL satellites. (C) R. Diehl
This demonstrates that the synthesis of new elements is an on-going process, continuously changing the element budget and distribution in our universe.

The sequence of nuclear reactions that develop during supernova explosions involve many short-lived nuclei far beyond the limits of nuclear stability. A study of these reactions and of the nuclei along the reaction path provides fundamental insight into the nature of these processes, the rapid timescale and dynamics of the explosion, the associated energy release and, of course, the synthesis of new elements. While experimental techniques are being developed now, the next-generation radioactive beam facilities, such as FAIR and FRIB, will generate the intensity necessary to produce key nuclei and to determine their properties. This will provide crucial input constraining the conditions during the explosion and will help to reliably model such events.

\section{The origin of the elements}

Recent years have witnessed impressive advances in the understanding of the microscopic processes that initiate and drive the evolution of the elements, through multiple sites, over the history of our universe. Light with its spectroscopic characteristics is the main signature for mapping and analyzing this evolution. The spectroscopy of stellar light with high resolution ground- and space-based telescopes indicates a gradual growth in the number of elements and in the increase of their abundances with time, confirming their continuous production in stars. The detailed structure of the elemental abundance distribution reflects the structure of nuclei participating in the various nucleosynthesis processes. These elements that have been built in the past are not only in stars, but also provide the chemical building stones of our planets, our earth, and ourselves. Carl Sagan summarized this remarkable development: "We are made of star stuff [10]". The observation and analysis of stellar light has confirmed this statement.

\section{About the Authors}

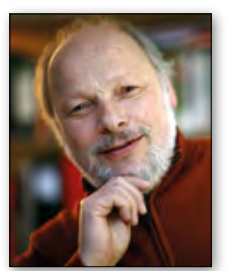

Michael Wiescher is an experimental nuclear physicist and the Director of the Nuclear Science Laboratory at the University of Notre Dame, USA. Previously he held positions at the University of Mainz, the California Institute of Technology and at the Ohio State University. He has been the founding Director of the Joint Institute for Nuclear Astrophysics and is the recipient of the 2003 APS Bethe Prize.

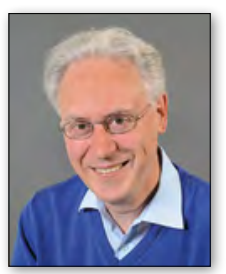

Karlheinz Langanke serves presently as Director of the GSI Helmholtzzentrum für Schwerionenforschung and is Professor at the Technical University of Darmstadt, working in theoretical nuclear astrophysics. Previously he held positions at the University of Aarhus in Denmark and at the California Institute of Technology. He is the recipient of the 2012 EPS Lise-Meitner Prize.

\section{References}

[1] E. G. Adelberger et al., Rev. Mod. Phys. 83, 195 (2011)

[2] E. Gawiser and J. Silk, Phys. Rep. 333, 245 (2000)

[3] C. C. Joggerst, S. E. Woosley and A. Heger, Astrophys. J. 693, 1780 (2009)

[4] S. C. Keller et al., Nature 506, 463 (2014)

[5] J. Cowan and C. Sneden, Nature 440, 1151 (2006)

[6] F.-K. Thielemann et al., Progr. Part. Nucl. Phys. 66, 346 (2011)

[7] M. Wiescher, F. Käppeler and K. Langanke, Ann. Rev. Astron. Astrophys. 50, 165 (2012)

[8] H.-Th. Janka et al., Phys. Rep. 442, 38 (2007)

[9] R. Diehl, N. Prantzos and P. von Ballmoos, Nucl. Phys. A 777, 70 (2006)

[10] C. Sagan, Cosmos (Random House, 1980) 